\title{
General Retinal Vessel Segmentation Using Regularization-based Multi-concavity Modeling
}

\author{
Benson S. Y. Lam, Member, IEEE, Yongsheng Gao, Senior Member, IEEE, Alan Wee-Chung Liew, \\ Senior Member, IEEE
}

\begin{abstract}
Detecting blood vessels in retinal images with the presence of bright and dark lesions is a challenging unsolved problem. In this paper, a novel multi-concavity modeling approach is proposed to handle both healthy and unhealthy retinas simultaneously. The differentiable concavity measure is proposed to handle bright lesions in a perceptive space. The lineshape concavity measure is proposed to remove dark lesions which have an intensity structure different from the line-shaped vessels in a retina. The locally normalized concavity measure is designed to deal with unevenly distributed noise due to the spherical intensity variation in a retinal image. These concavity measures are combined together according to their statistical distributions to detect vessels in general retinal images. Very encouraging experimental results demonstrate that the proposed method consistently yields the best performance over existing state-of-the-art methods on the abnormal retinas and its accuracy outperforms the human observer, which has not been achieved by any of the state-of-the-art benchmark methods. Most importantly, unlike existing methods, the proposed method shows very attractive performances not only on healthy retinas but also on a mixture of healthy and pathological retinas.
\end{abstract}

Index Terms - Retinal vessel segmentation, retina image, multiconcavity modeling, perceptive transform, regularization.

\section{INTRODUCTION}

$\mathrm{B}$ right and dark lesions are the symptoms of retinal diseases arising from diabetic retinopathy, hypertensive retinopathy, solar retinopathy, retinal vein or artery occlusion, etc. [1-4]. Retinopathies can progress to blindness or severe loss of vision. However, half of the blindness can be prevented by regular screening and timely treatment [5]. This is a labor intensive process, which requires clinical experts to examine a large number of retinas. Several retina centers such as the Joslin Vision Network and Inoveon Corp. have shown that digital photography is an excellent tool for analyzing retina [6, 7] and computer algorithms are being developed for

Manuscript received on July 21, 2009. This research is supported by the Australian Research Council (ARC) under Discovery Grants DP0451091 and DP0877929. B. Lam is with Griffith School of Engineering, Griffith University, Australia (e-mail: b.lam@griffith.edu.au). Y. Gao is with Griffith School of Engineering, Griffith University, Australia and National ICT Australia, Queensland Research Laboratory (e-mails: yongsheng.gao@griffith.edu.au, yongsheng.gao@nicta.com.au). A. Liew is with the School of Information and Communication Technology, Griffith University, Australia and National ICT Australia, Queensland Research Laboratory (e-mail: a.liew@griffith.edu.au). automatic retinal image analysis [8-23]. Blood vessel is one of the most important features in retina for detecting retinal vein occlusion [24], for grading the tortuosity for hypertension [25] and for early diagnosis of glaucoma [15]. The segmentation of blood vessels is an important preprocessing step for the detection of bright and dark lesions [26-30]. However, most existing blood vessel segmentation algorithms assume that the input retina is healthy and free of bright and dark lesions. The presence of lesions can significantly degrade their performances and even make them useless.

Concavity in the intensity profile is one of the most important image properties in blood vessel segmentation and most existing methods assume that the intensity profile of a vessel has an elongated concave structure within a smooth and clean non-vessel region. However, when bright and dark lesions exist, the non-vessel region is not smooth anymore. Figures 1 (a) and (c) show retinal images having bright lesions and dark lesions respectively. Their cross sections intensity profiles are given in Figures 1(b) and (d) respectively. These lesions violate the basic assumption of existing methods and lead to a large number of false positives.

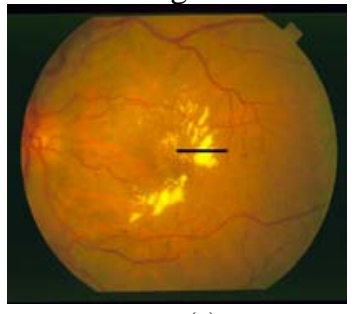

(a)

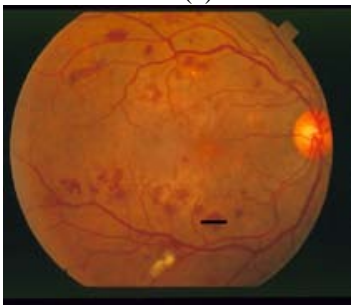

(c)

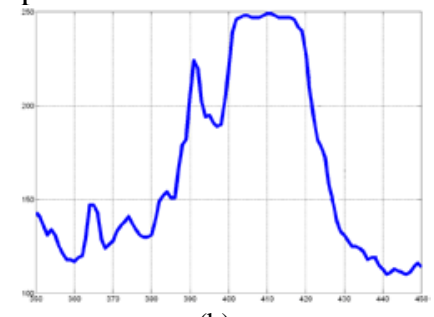

(b)

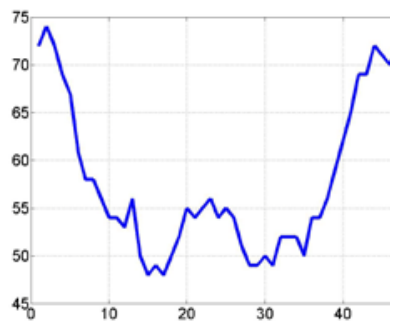

(d)
Figure 1. (a) An abnormal retina with bright lesions. (b) Cross-section intensity profile of the abnormal region marked in (a). (c) An abnormal retina with dark lesions. (d) Cross-section intensity profile of the abnormal region marked in (c).

Existing retinal vessel segmentation approaches can be classified into three categories: unsupervised methods, supervised learning methods and pathological retina based methods. For unsupervised methods, Chaudhuri et al. [31] proposed a template matching approach to measure the degree 
of similarity between a given elongated inverse Gaussian template and the vessels. Hoover et al. [32] enhanced the above method by applying effective noise removal techniques. Jiang and Mojon [33] proposed an adaptive thresholding technique which partitions the input image into a finite number of levels according to pixel intensities. In each level, the vessels are extracted using the intensity, angle and length information based on a pre-defined elongated concave structure. Ricci and Perfetti [34, 35] proposed a fast and robust template matching algorithm which measures the output difference between a line filter and an averaging filter. Tolis et $a l$. [36] proposed a tracking approach in which seed points are grown according to pre-defined rules to extract all concave regions as vessels. These methods work well on healthy retinas but fail to perform on abnormal ones because all non-vessel regions are assumed to be smooth, which is not true when bright and dark lesions exist.

In contrast to unsupervised methods, supervised learning methods adopt human segmentation results to define the concave intensity structure of retinal vessels and the smooth characteristic of the non-vessel regions. Most state-of-the-arts supervised learning methods handle only healthy retina and reported that retina having lesions are challenging future work [37-39]. Staal et al. [37,38] is one of the very first research groups using the supervised learning strategy for vessel segmentation. Numerous features are used for training a classifier to classify pixels as either vessel or non-vessel. The method yields very good results on healthy retinal images. However, many of these features, such as the Laplacian of Gaussian features which detect concavity intensity profile in retinal vessels, require a high degree of smoothness in the nonvessel region. If a bright lesion is present, the steep intensity transition pattern of the lesion will also be treated as vessels, producing a large number of artifacts. Soares et al. [39] proposed a Gabor feature based supervised learning method for vessel segmentation. They adopted the Gabor features which consider the low frequency elongated structure in the intensity profile of the retinal vessels in the training process. This approach has excellent performance on healthy retinas. However, due to the detection of low frequency features, the vessels located near the high frequency bright lesions are discarded.

Pathological retina based methods are the only class in which pathology in retinas is considered during vessel detection. There are few publications in this category and they only handle bright lesions. Mendonça and Campilho [40] adopted different color space for different retinal images and used the L*a*b* color space to handle bright lesions. Their method produces better results on the pathological retina than most of the existing algorithms. Recently, Lam and Yan [41] proposed a divergence vector field approach to handle the bright lesions by measuring the smoothness of the non-vessel regions in different orientations. Although the method can handle bright lesions, a thresholding technique is needed for artifacts removal. A large threshold leads to more artifacts removal near the bright lesions but also results in many normal vessels being incorrectly removed.

Clearly, there is a need to develop new vessel segmentation methods that are effective for both normal and pathological retinas with bright and dark lesions. In this paper, we present an effective algorithm that, for the first time, is able to handle both normal and pathological retinas with bright and dark lesions simultaneously. Three different concavity measures are proposed to detect blood vessels and each of these measures is designed for addressing the negative impact produced by the lesions for identifying the normal vessels. Using Weber's law, we propose a perceptive transform to model human visual perception in retinal image analysis. As the bright lesion has a steep intensity transition pattern, measuring the degree of concavity based on differentiability (Section II) can effectively distinguish the bright lesions from the vessels and non-vessels. Since dark lesions have an irregular shape intensity structure while blood vessels have a line-shape intensity structure, a line-shape concavity detection method (Section III) is developed to prune the dark lesions while keeping the lineshape blood vessels. As retinal image has a spherical intensity variation, the relative intensities of noise in different parts of the retinal image are different. A locally normalized concavity detection method (Section III) is proposed to normalize the strengths of noise removal in different regions. Finally, these concavity measures are combined according to their statistical and geometrical properties (Sections IV and V). A flow-chart of the proposed method is given in Figure 2. The effectiveness and robustness of the proposed method are evaluated on two publicly available databases (STARE and DRIVE) and are compared with the state-of-the-art methods.

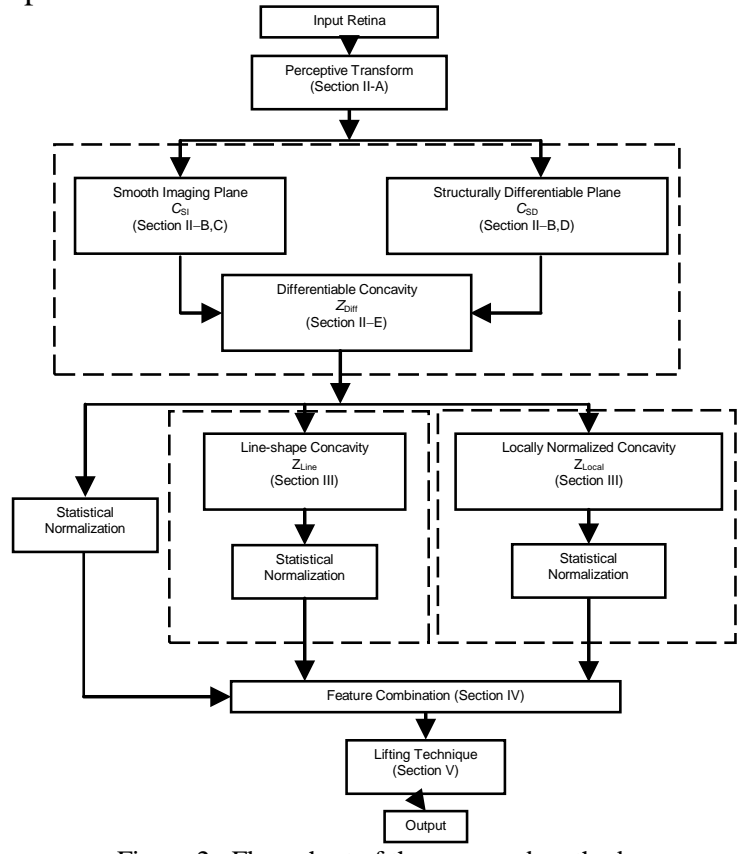

Figure 2. Flow-chart of the proposed method.

The rest of the paper is organized as follows. Section II describes the perceptive differentiable concavity measure to handle the bright lesions. Section III presents the line-shape concavity measure to handle dark lesions and the locally normalized concavity measure for noise removal. Section IV describes how the features obtained from these concavity measures are combined and Section $\mathrm{V}$ describes the lifting technique for optimizing the regularized solution toward the ideal vessel shape. The experimental results are given in 
Section VI. Finally, the conclusions are given in Section VII.

\section{PERCEPTIVE DIFFERENTIABLE CONCAVITY}

In this section, a differentiable concavity measure $\mathrm{Z}_{\mathrm{Diff}}(\mathrm{z})$ is designed to robustly detect retinal blood vessels in the presence of bright lesions. This measure is constructed on our proposed perceptive space, which mimics human perception in retinal image analysis.

\section{A. Perceptive Transform}

Ernst Weber [42] examined the relationship between the physical intensity magnitude and its perceptive intensity. He observed that the just noticeable change of physical magnitude by human perception can be described by the equation

$$
\frac{\delta f(z)}{f(z)}=k^{*}
$$

where $f(z)$ is the physical magnitude at $z, \delta f(z)$ is its rate of change and $k^{*}$ is the Weber's constant. If the quantity on the left hand side of the equation is smaller than $k^{*}$ at $\mathrm{z}=\mathrm{z}^{*}$, human cannot notice the change of the physical magnitude.

Given a perceptive intensity $v^{0}$, the rate of change of the perceptive intensity and the physical magnitude is related by [43]

$$
\frac{\delta f(z)}{f(z)}=k \times \delta v^{0}(z)
$$

Obviously, if $\delta v^{0}$ is small, it is hard to notice the change of the physical magnitude, and vice versa. In this research, the physical magnitude is the intensity of a two dimensional image and the rate of change $\delta f(z)$ is defined as gradient of the physical magnitude. Transforming an input image $I^{0}$ to its perceptive intensity $v^{0}$ can be achieved by the following theorem.

Theorem 1: Assume $v^{0}(0,0)=0$ and $f(0,0)=1$. Given the physical magnitude function $f(x, y)$ in Equation (2), when the rate of change $\delta f(x, y)$ at $(x, y)$ is small enough, the perceptive intensity $v^{0}(x, y)$ is expressed as $v^{0}(x, y)=\ln (f(x, y)) / k$.

Proof: In Equation (2), when the rate of change $\delta f(x, y)$ at $(x, y)$ is small enough, the gradient operator $\nabla$ can be used as an estimate of $\delta$. That is, Equation (2) can be rewritten as

$$
\frac{\nabla f(x, y)}{f(x, y)}=k\left(\nabla v^{0}(x, y)\right) \text {. }
$$

The $x$ and $y$ components of the gradient operator is separable. At $y=y_{0}$, the $x$-component of Equation (3) becomes

$$
\frac{d_{x} f\left(x, y_{0}\right)}{f\left(x, y_{0}\right)}=k\left(d_{x} v^{0}\left(x, y_{0}\right)\right) \text {. }
$$

By integrating both sides of the above equation from $x=0$ to $x$ $=x_{0}$, we have

$$
\ln \left(f\left(x, y_{0}\right)\right)-\ln \left(f\left(0, y_{0}\right)\right)=k\left(v^{0}\left(x, y_{0}\right)-v^{0}\left(0, y_{0}\right)\right)
$$

where $\ln (\bullet)$ is the natural logarithm. Similarly, for the $y$ - component, we have

$$
\ln \left(f\left(x_{0}, y\right)\right)-\ln \left(f\left(x_{0}, 0\right)\right)=k\left(v^{0}\left(x_{0}, y\right)-v^{0}\left(x_{0}, 0\right)\right)
$$

By taking $y_{0}=y$ in Equation (5) and $x_{0}=0$ in Equation (6) and summing up these two equations, we get

$$
\ln (f(x, y))-\ln (f(0,0))=k\left(v^{0}(x, y)-v^{0}(0,0)\right)
$$

As $v^{0}(0,0)=0$ and $f(0,0)=1$, we have

$$
\ln (f(x, y))=k\left(v^{0}(x, y)\right)
$$

There are two assumptions $\left(v^{0}(0,0)=0\right.$ and $f(0,0)=$ 1 ) in the above theorem. If we take $f(z)=1+I^{0}(z)$ with $z$ $=(x, y)$, the assumptions will be satisfied automatically. In a retinal image, the pixels along the border of the image (outside the field of view (FOV)) has zero intensity, that is, $I^{0}(0)=0$. This means that $f(0)=1$ and $v^{0}(0)=\ln (1+0)=0$. Thus, the equation transforming the input image $I^{0}$ to the perceptive image $v^{0}$ can be expressed as

$$
v^{0}(z)=\ln \left(1+I^{0}(z)\right) / k
$$

where $k$ is a constant. The constant $k$ is taken to be one in our experiments. A different value of $k$ does not affect the segmentation result as $k$ can be digested by the normalization process and the user-defined parameter of the TV model. More details will be given in Section II-E. Non-retinal images (or a cropped retinal image) may not have a zero intensity pixel or the zero intensity pixels may not be located at the border of the image. If that is the case, the transform will become

$$
v^{0}(z)=(\ln f(z)-\ln f(0)) / k .
$$

The unknown parameter $\ln f(0)$ can be ignored as the constructions of the two planes $\mathrm{C}_{\mathrm{SI}}$ and $\mathrm{C}_{\mathrm{SD}}$ are invariant under translation to the perceptive intensity as will be shown in Section II-E. Now that a transform to obtain a perceptive intensity has been formulated, we apply this technique to analyze the concavity profile with the presence of bright lesions.

\section{B. Bright Lesion}

Existing retinal vessel segmentation methods assume vessels in a retina have a concave shape intensity profile within a smooth and clean non-vessel region. The non-smooth bright lesions having a steep intensity transition pattern can therefore be wrongly classified as vessels. Figure 1(a) shows an example of bright lesions with its cross-section intensity profile shown in Figure 1(b). Figure 3 is the result of a well-known concavity detection algorithm [44] which computes the second order directional derivatives in a multi-scale manner. Due to the steep intensity transition pattern of the bright lesions, the method falsely classifies the non-smooth pixels as vessels and a ringing effect is produced in the lesion regions.

To deal with the negative effect of the steep intensity transition pattern in the bright lesions, a differentiable concavity measure is proposed to describe vessels. The input retina is first transformed into the proposed perceptive space (see Section II-A). Then, the problem of ringing effect is 
resolved by constructing two planes using regularizations: smooth imaging plane $C_{\mathrm{SI}}$ (see Section $\mathrm{II}-\mathrm{C}$ ) and structurally differentiable plane $C_{\mathrm{SD}}$ (see Section II-D). The smooth imaging plane classifies the smooth regions as non-vessel region while the structurally differentiable plane classifies the non-smooth steep intensity transition pattern of bright lesions as non-vessel region. Together, these two planes can categorize the bright lesions and the smooth background region as non-vessel and the smooth concave regions as vessels. In the next section, we will first introduce the concept of smooth imaging plane $\left(C_{\mathrm{SI}}\right)$.

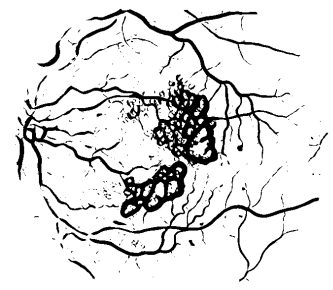

Figure 3. Vessel segmentation result using the multi-scale method [44] with $(f p r, t p r)=(0.0943,0.7225)$ to the retinal image in Figure 1(a).

\section{Smooth Imaging Plane}

The smooth imaging plane $C_{\mathrm{SI}}$ is constructed by measuring the concavity strength in a low perceptively noticeable environment. In the perceptive domain, the degree of noticeable change is given by Equation (2), whose right hand side is the gradient of the perceptive image $v^{0}(\mathrm{x}, \mathrm{y})$. By minimizing this term, the degree of noticeable change is minimized and pixels with non-noticeable change are smoothed out. To preserve the detailed information of the input retina, a fitting term $\left(\left\|v-v^{0}\right\|^{2}\right)$ which measures the degree of similarity of the solution $v(\mathrm{x}, \mathrm{y})$ and the input image $v^{0}(\mathrm{x}, \mathrm{y})$ is applied. The regularized solution $v(\mathrm{x}, \mathrm{y})$ can be obtained by minimizing the following objective function [45, 46]

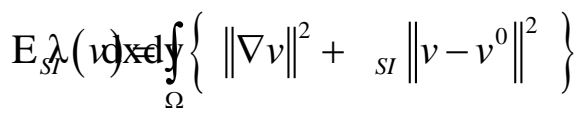

where $\Omega$ is the domain, $\lambda_{\mathrm{SI}}$ is a user-defined parameter. $\mathrm{E}_{\mathrm{SI}}(v)$ is the Mumford-Shah (MS) model $[45,46]$, which consists of two terms, the regularization term and the fitting term measuring the difference between the solution and the input. Using the calculus of variation, the minimum $v^{\text {SI }}$ of the objective function $\mathrm{E}_{\mathrm{SI}}(v)$ satisfies the following equation

$$
-\nabla^{2} v^{S I}+\lambda_{S I}\left(v^{S I}-v^{0}\right)=0 \text {. }
$$

In this equation, the isotropic Laplacian operator $\nabla^{2}$ is used as regularization and this equation is solved by the numerical scheme given in the Appendix A, in which the regularization is applied to both the magnitude and the orientation of the solution $v^{\text {SI }}$. It behaves like a diffusion process smoothing out noise.

The strength in the smooth imaging plane $C_{\mathrm{SI}}$ is obtained by computing the squared maximum principal curvature $m^{\text {SI }}$ [44]. The principal curvature $\mathrm{d}_{\theta \theta} v^{\mathrm{SI}}$ with respect to a given principal direction $\theta$ is defined as [44]

$$
\mathrm{d}_{\theta \theta} v^{\mathrm{SI}}=\left[\begin{array}{ll}
\cos \theta & \sin \theta
\end{array}\right]\left[\begin{array}{ll}
v_{x x}^{\mathrm{SI}} & v_{x y}^{\mathrm{SI}} \\
v_{x y}^{\mathrm{SI}} & v_{y y}^{\mathrm{SI}}
\end{array}\right]\left[\begin{array}{c}
\cos \theta \\
\sin \theta
\end{array}\right] .
$$

The subscripts in Equation (13) denote the partial derivatives of $v^{\mathrm{SI}}$ along the $x$ and $y$ coordinates. Then, $C_{\mathrm{SI}}=m^{\mathrm{SI}}$ can be obtained by the following equation [44]

$$
C_{S I}=m^{\mathrm{SI}}=\left(\frac{v_{x x}^{\mathrm{SI}}+v_{y y}^{\mathrm{SI}}+\sqrt{\left(v_{x x}^{\mathrm{SI}}-v_{y y}^{\mathrm{SI}}\right)^{2}+4\left(v_{x y}^{\mathrm{SI}}\right)^{2}}}{2}\right)^{2}
$$

which is the squared maximum eigenvalue of the Hessian matrix given in Equation (13). Figure 4 shows the segmentation result in the smooth imaging plane for the retina image of Figure 1(a). Compare to the segmentation result in Figure 3, it can be seen that $C_{\mathrm{SI}}$ is able to reduce the artifacts due to the bright lesions (fpr reduced by 0.0292) while revealing more vessels (tpr increased by 0.0417 ). To handle the artifacts near the bright lesions, a structurally differentiable plane is proposed in the next sub-section.

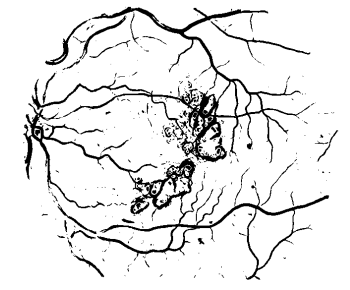

Figure 4. Segmentation result in $C_{\mathrm{SI}}$ at $(\mathrm{fpr}, \mathrm{tpr})=(0.0651,0.7642)$.

\section{Structurally Differentiable Plane}

The concept of constructing the structurally differentiable plane $C_{\mathrm{SD}}$ is to perform regularization to the input $v^{0}$ so that the output ignores the contributions of all steep intensity transition patterns while revealing the smooth concave-shape vessels. That is, $C_{\mathrm{SD}}$ should give low responses to highly discontinuous signals such as bright lesions and high responses to smoother signals such as vessels. The negation of $C_{\mathrm{SD}}$ can be obtained by minimizing the total variation (TV) model [46, 47] which preserves sharp edges (such as bright lesions) of the input image while performs strong smoothing to the smoother signals (such as vessels).

The TV model is given by

$$
\mathrm{E}_{S D}(v)=\int_{\Omega}\left\{\|\nabla v\|_{\tau}+\frac{\lambda_{S D}}{2}\left\|v-v^{0}\right\|^{2}\right\} \mathrm{dxdy}
$$

where $\|\nabla v\|_{\tau}=\sqrt{\tau^{2}+\|\nabla v\|^{2}}$ and $\lambda_{\mathrm{SD}}$ is a user-defined constant. $\tau$ is a parameter to avoid the non-differentiability at $v$ $=0$ and we take $\tau=10^{-4}$ in all the experiments. The key difference between Equation (15) and Equation (11) is in the regularization term. The regularization in the MS model is the square of the gradient amplitude while the regularization in the TV model does not have a square. Using the calculus of variation, the minimum $v^{\mathrm{SD}}$ of the objective function of Equation (15) satisfies

$$
\nabla \cdot \frac{\nabla v^{S D}}{\left\|\nabla v^{S D}\right\|_{\tau}}+\lambda_{S D}\left(v^{0}-v^{S D}\right)=0
$$


The solution $v^{\mathrm{SD}}$ is computed by the scheme given in the Appendix A. The regularization computes the curvature (the rate of change of orientation) of the solution $v^{\mathrm{SD}}$. If a pixel and its neighborhood have a steep intensity transition pattern, $\frac{1}{\left\|\nabla v^{S D}\right\|_{\tau}}$ will be very small and the regularization term $\left(\nabla \cdot \frac{\nabla v^{S D}}{\left\|\nabla v^{S D}\right\|_{\tau}}\right)$ to this pixel is greatly reduced, and thus the solution $v^{S D}$ of the pixel will be equal to its original input $v^{0}$. Compare to the highly discontinuous steep intensity transition pattern in bright lesions, a vessel in a retina is much smoother. Because of this smoothness, the regularization term in the vessel region is much stronger than that in the non-smooth bright lesions. Figure 5(a) shows the output of the TV model. The bright lesions are preserved while other concave blood vessels are smoothed out. By subtracting the original input $v^{0}$ from $v^{\mathrm{SD}}$, the structurally differentiable plane $C_{\mathrm{SD}}$ (i.e. $C_{\mathrm{SD}}=$ $v^{\mathrm{SD}}-v^{0}$ ) could be obtained. If $v^{\mathrm{SD}}$ has a large value (such as bright lesions), $C_{\mathrm{SD}}=v^{\mathrm{SD}}-v^{0}$ will return a low value and vice versa. Figure 5(b) shows the vessel segmentation result in the structurally differentiable plane, in which no ringing effect is produced. In the next section, we combine this structurally differentiable plane with the smooth imaging plane to form the differentiable concavity measure, which is robust for detecting blood vessels with the presence of bright lesions.

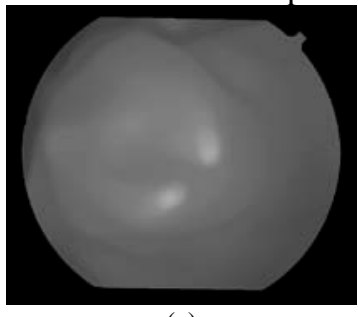

(a)

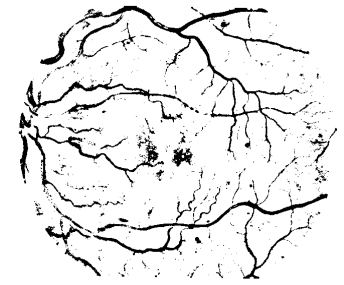

(b)
Figure 5. Segmentation in the structurally differentiable plane. (a) The output of the TV model. (b)The vessel segmentation result in $C_{\mathrm{SD}}$ at $(\mathrm{fpr}, \mathrm{tpr})=$ $(0.0389,0.7124)$.

\section{E. Differentiable Concavity}

The two imaging planes are combined to form a differentiable concavity measure. It is observed that a retinal pixel belongs to a non-vessel region when both planes have low strengths. It is also obvious that a pixel belongs to a vessel when both planes show high responses. For the case of high strength in $C_{\mathrm{SI}}$ and low strength in $C_{\mathrm{SD}}$, a pixel is observed as the ringing effect due to its high response to the smooth plane but not to the structurally differentiable plane. As noise can have a concave intensity profile but is not smooth, a pixel is considered as noise when it has low strength in $C_{\mathrm{SI}}$ and high strength in $C_{\mathrm{SD}}$. Based on the above observations (summarized in Table 1), a new differentiable concavity measure $Z_{\text {Diff }}$ is defined as the conditional probability

$$
Z_{\text {Diff }}(z)=\operatorname{Pr}\left(C_{S D}(z), C_{S I}(z) \mid I^{0}(z)\right)
$$

where $I^{0}$ is the input image. The differentiable concavity measure $Z_{\text {Diff }}$ inherits the strengths of both planes. When the two image planes $C_{\mathrm{SI}}, C_{\mathrm{SD}}$ are independent, the above function can be written as

$$
Z_{\text {Diff }}(z)=\operatorname{Pr}\left(C_{S D}(z) \mid I^{0}(z)\right) \operatorname{Pr}\left(C_{S I}(z) \mid I^{0}(z)\right) \text {. }
$$

The independence can be justified as follows. The maximum eigenvalue of the Hessian matrix is used to determine the strength of $C_{\mathrm{SI}}$ (Equation (14)) while the curvature $\left(\nabla \cdot \frac{\nabla v^{S D}}{\left\|\nabla v^{S D}\right\|_{\tau}}\right.$ in Equation (16)) is used to construct $C_{\mathrm{SD}}$. The curvature here only involves the rate of change of orientation and does not involve other variables. Any change of orientation does not influence the magnitude of the maximum eigenvalue of a Hessian matrix. Thus, $C_{\mathrm{SD}}$ and $C_{\mathrm{SI}}$ are orthogonal to each other.

Table 1. The combined effects of the two planes

\begin{tabular}{|c|c|c|}
\hline Image planes & Strengths & Effect \\
\hline$\left(\mathrm{C}_{\mathrm{SI}}, \mathrm{C}_{\mathrm{SD}}\right)$ & (low, low) & Non-vessel \\
\hline$\left(\mathrm{C}_{\mathrm{SI}}, \mathrm{C}_{\mathrm{SD}}\right)$ & (high, high) & Vessel \\
\hline$\left(\mathrm{C}_{\mathrm{SI}}, \mathrm{C}_{\mathrm{SD}}\right)$ & (high, low) & Ringing effect \\
\hline$\left(\mathrm{C}_{\mathrm{SI}}, \mathrm{C}_{\mathrm{SD}}\right)$ & (low, high) & Noise \\
\hline
\end{tabular}

The two probability maps $\operatorname{Pr}\left(C_{\mathrm{SI}} \mid I^{0}\right)$ and $\operatorname{Pr}\left(C_{\mathrm{SD}} \mid I^{0}\right)$ are of the same size as the original input image, in which each pixel is now of a probability value. By Equation (14), squared maximum eigenvalue is used and $C_{\mathrm{SI}}$ must be positive. The computation of the probability map $\operatorname{Pr}\left(C_{\mathrm{SI}} \mid I^{0}\right)$ can be obtained by normalizing $C_{\mathrm{SI}}$ as

$$
\operatorname{Pr}\left(C_{S I}(z) \mid I^{0}(z)\right)=\frac{C_{S I}(z)}{n_{\text {constant }}}
$$

where $n_{\text {constant }}$ is the normalization constant and is equal to the summation of $C_{\mathrm{SI}}$ over the field of view (FOV). In retinal image analysis, FOV is defined as the circular retinal region located in the middle of a rectangular image and the region outside is the dark non-retina background. We now discuss the effect of the two unknown parameters $\ln f(0)$ and $k$ in Equation (10) to the construction of $C_{\mathrm{SI}}$. Obviously, the objective function Equation (11) has a linear relationship between the input $v^{0}$ and the output $v^{\text {SI }}$. That is, if $v^{\text {SI }}$ is a minimum of $\mathrm{E}_{\mathrm{SI}}(v)$ with input $v^{0}, \mathrm{~s} v^{\mathrm{SI}}+\mathrm{b}$ is the minimum of $\mathrm{E}_{\mathrm{SI}}(v)$ with input $\mathrm{s} v^{0}+\mathrm{b}$. As $C_{\mathrm{SI}}$ is obtained by making use of derivatives (Equation (13)), $C_{\mathrm{SI}}$ is invariant under a translation of the perceptive intensity of $v^{0}$. Moreover, the use of normalization constant given in Equation (19) makes the probability $\operatorname{Pr}\left(C_{\mathrm{SI}} \mid I^{0}\right)$ invariant under a scaling of the perceptive intensity of $v^{0}$. Thus, the two parameters $\ln f(0)$ and $k$ given in Equation (10) can be ignored. By Equation (16), the output $v^{\mathrm{SD}}$ can have either a larger or smaller value than the original input $v^{0}$ and $C_{\mathrm{SD}}=v^{\mathrm{SD}}-v^{0}$ can be negative. The construction of the probability map $\operatorname{Pr}\left(C_{\mathrm{SD}} I^{0}\right)$ can be obtained by discarding the negative part of $C_{\mathrm{SD}}$

$$
\operatorname{Pr}\left(C_{S D}(z) \mid I^{0}(z)\right)=\left\{\begin{array}{cl}
\frac{C_{S D}(z)}{d_{\text {constant }}} & \text { if } C_{S D}(z)>0 \\
0 & \text { otherwise }
\end{array}\right.
$$

where $d_{\text {constant }}$ is the normalization constant and is equal to the summation of $C_{\mathrm{SD}}$ over FOV. Next, we discuss the effect of 
$\ln f(0)$ and $k$ to the construction of $\mathrm{C}_{\mathrm{SD}}$. The optimization function in Equation (16) has the following translation relationship: if $v^{\mathrm{SD}}$ is a minimum of $\mathrm{E}_{\mathrm{SD}}(v)$ with input $v^{0}, v^{\mathrm{SD}}+\mathrm{b}$ is the minimum of $\mathrm{E}_{\mathrm{SD}}(v)$ with input $v^{0}+\mathrm{b}$. The construction of $C_{\mathrm{SD}}$ is given by v $\mathrm{v}^{\mathrm{SD}}-v^{0}$, which implies $C_{\mathrm{SD}}$ is invariant under a translation of $v^{0}$. Hence, the translation parameters $\ln f(0)$ can be ignored. Regarding the scaling parameter $k$ in Equation

(10), note that the regularization term $\nabla \cdot \frac{\nabla v^{S D}}{\left\|\nabla v^{S D}\right\|_{\tau}}$ Equation (16) is scaling invariant for the variable $v^{\mathrm{SD}}$. That is, if $v_{\mathrm{SD}}$ is a minimum of $\mathrm{E}_{\mathrm{SD}}(v)$ with input $v^{0}$ and user-defined parameter $\lambda_{\mathrm{SD}},\left(v_{\mathrm{SD}}\right) / k$ is the minimum of $\mathrm{E}_{\mathrm{SD}}(v)$ with input $\left(v^{0}\right) / k$ and $k \lambda_{\mathrm{SD}}$. By setting $k \lambda_{\mathrm{SD}}$ as a user-defined parameter, the scaling parameter $k$ can be ignored. Figure 6(a) shows the segmentation result using the differentiable concavity measure $Z_{\text {Diff. }}$ It can be seen that the ringing effect around the bright lesions are largely eliminated and the effect of noise is reduced in the non-vessel region. In the next section, we will propose a locally normalized concavity measure to further remove the noise around the bright lesions and at the non-vessel region. Moreover, a line-shape concavity is proposed to detect vessels with the presence of dark lesions.

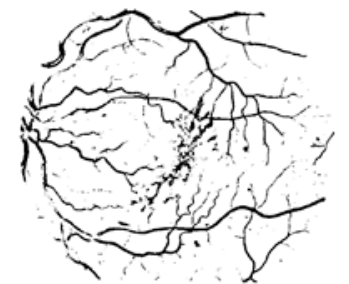

(a)

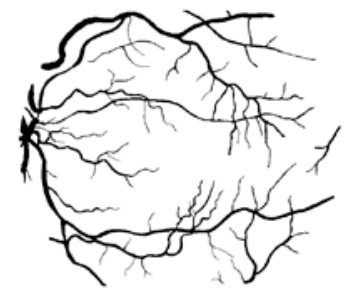

(b)
Figure 6. (a) Segmentation result of using the differentiable concavity measure $Z_{\text {Diff }}$ at $(f p r, t p r)=(0.0318,0.7073)$. (b) Ground truth .

\section{LINE-SHAPE CONCAVITY AND LOCALLY NORMALIZED CONCAVITY}

Other than bright lesions, a pathological retina can also appear in the form of dark lesions, e.g., due to hemorrhages, having concavity intensity profile similar to vessels. Figure 7(a) shows an example retina with dark lesions. Due to the concavity intensity profile of dark lesions, many vessel detectors give a large response to the dark lesions. Figure 7(b) is an example segmentation result showing many false positive dots which are incorrectly detected as vessels. To distinguish the dark lesions from the vessels, a line-shape concavity measure $Z_{\text {Line }}$ based on the geometric structural difference of the two regions is proposed.

The vessel has an intensity profile uniformly distributed along a line with direction $\Theta$ while the dark lesion has an intensity profile uniformly distributed on an irregular patch. Likelihood ratio is used to model this shape difference. The line-shape concavity measure $Z_{\text {Line }}$ is defined as the likelihood ratio

$$
Z_{\text {Line }}(z)=\frac{\sup \left\{\operatorname{Pr}\left(Z_{\text {Diff }} \mid S\right): S \in \Theta_{0}\right\}}{\sup \left\{\operatorname{Pr}\left(Z_{\text {Diff }} \mid S\right): S \in \Theta_{1}\right\}}
$$

where sup is the supremum, $\operatorname{Pr}\left(Z_{\text {Diff }} \mid S\right)$ is the conditional probability $Z_{\text {Diff }}(\mathbf{z})$ given the shape $S$. This likelihood ratio returns a large response value at pixel $z$ if $Z_{\text {Diff }}(z)$ contains the shape pattern of $\Theta_{0}$ more strongly than that of $\Theta_{1}$. Otherwise, it gives a small value. $\Theta_{0}$ is the space of line shapes containing a collection of lines with different orientations, which are taken from 0 to $\pi$ with an interval of $\pi / 12$. This kind of line shapes is shown to be robust for detecting linear structure [34, $35,48] . \Theta_{1}$ is the space of square shapes. The probability $\operatorname{Pr}\left(Z_{\text {Diff }} \mid S\right)$ is computed by applying a filter $S$ to $Z_{\text {Diff }}(\mathrm{z})$ in the shape space $\Theta_{0}$ or $\Theta_{1}$. In $\Theta_{0}$, the set of line filters with window size 15 [34] is adopted. In $\Theta_{1}$, the square shape average filter with window size 15 [34] is used. Figure 7(d) shows the segmentation result of using the line-shape concavity measure, in which most of the false positives from using the $Z_{\text {Diff }}$ measure (Figure 7(c)) are removed. Figure 8(a) shows the segmentation result using the line-shape concavity measure for the retinal image of Figure 1(a) with bright lesions.

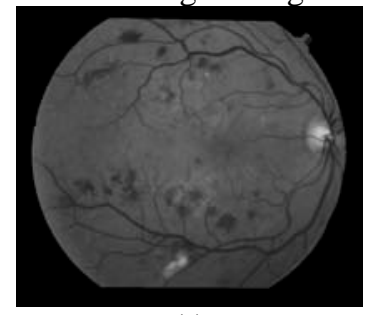

(a)

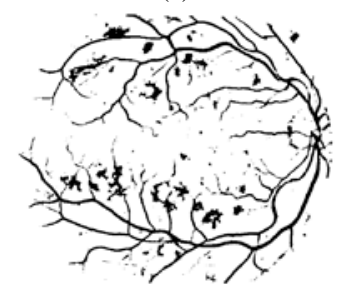

(c)

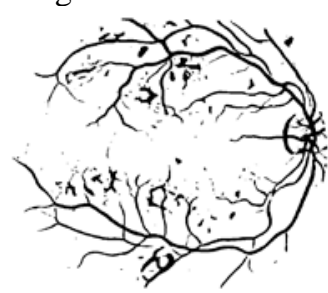

(b)

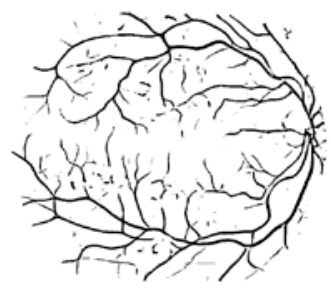

(d)

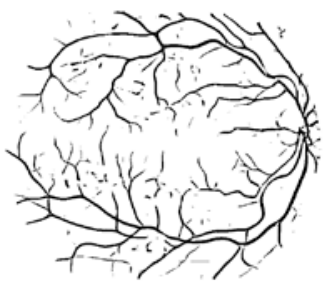

(e)

Figure 7. (a) A retina image having dark lesions. (b) Segmentation using the multi-scale method [44] at $(\mathrm{fpr}, \mathrm{tpr})=(0.0531,0.7640)$. (c) Segmentation using the differentiable concavity measure $Z_{\text {Diff }}$ at $(f p r, t p r)=(0.0553,0.7693)$.

(d) Segmentation using the line-shape concavity measure $Z_{\text {Line }}$ at $(f p r, t p r)=$ $(0.0270,0.7535)$. (e) Segmentation using the locally normalized concavity measure $Z_{\text {Local }}$ at $(\mathrm{fpr}, \mathrm{tpr})=(0.0262,0.7495)$.

Next, we discuss the noise problem. The shapes due to noise always appear as tiny dots and are very different from the vessels. Noise intensity also varies in different part of the retina, e.g. the noise intensities at the high intensity bright lesions are larger while they are smaller near the low intensity macula. To suppress the effect of noise, a locally normalized concavity measure $Z_{\text {Local }}$ is proposed based on the shape and local intensity information.

Line structure carries effective information to suppress noise and preserve the vessels. However, due to the varying intensity of noise in different parts of the retina, line information alone 
is not enough. To normalize this intensity variation in different regions, the original input intensity (which has the same intensity variation as noise) is embedded in the probability $\operatorname{Pr}\left(Z_{\text {Diff }} \mid S\right)$. The new probability is defined as

$$
\operatorname{Pr}_{\text {local }}\left(Z_{\text {Diff }} \mid S\right)=\frac{\operatorname{Pr}\left(Z_{\text {Diff }} \mid S\right)^{r}}{m_{\text {constant }}}
$$

where $r(\mathbf{z})=1 /\left(1+I^{0}(\mathbf{z})\right)$ and $m_{\text {constant }}$ is a normalization constant. The use of $1+I^{0}$ is to avoid division by zero when $I^{0}(\mathbf{z})=0$. The exponent $r(\mathbf{z})$ is inversely proportional to the input intensity. Hence, if the computation is made at the bright lesion, the high intensity noise value is suppressed by the high input intensity. If it is in the macula (a large concave region having low intensity value), the low intensity noise value is normalized by the low input intensity. Line-shape detection technique can then be used for noise removal using the following equation

$$
Z_{\text {Local }}(z)=\frac{\sup \left\{\operatorname{Pr}_{\text {local }}\left(Z_{\text {Diff }} \mid S\right): S \in \Theta_{0}\right\}}{\sup \left\{\operatorname{Pr}_{\text {local }}\left(Z_{\text {Diff }} \mid S\right): S \in \Theta_{1}\right\}}
$$

where $\Theta_{0}$ and $\Theta_{1}$ are defined the same as in Equation (21). Figure $7(\mathrm{e})$ and $8(\mathrm{~b})$ show results of $\mathrm{Z}_{\text {Local }}(\mathbf{z})$ on the retina images having bright and dark lesions, respectively. In Figure 8(b), the noise due to the bright lesions is completely removed obtaining a clean non-vessel region. Next, we combine all three concavity measures (differentiable concavity, line-shape concavity and locally normalized concavity) into one blood vessel segmentation system.

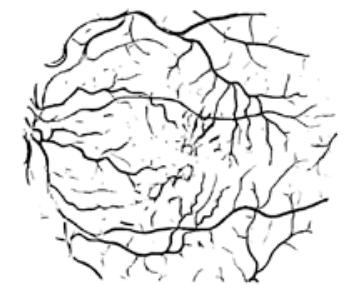

(a)

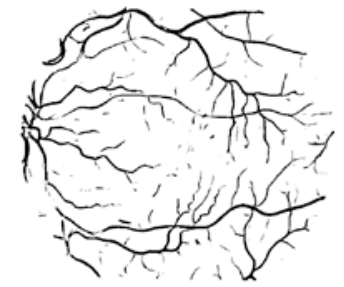

(b)
Figure 8. Segmentation results for the retinal image of Figure 1(a) having bright lesions using: (a) the line-shape concavity measure $Z_{\text {Line }}$ for $(\mathrm{fpr}, \mathrm{tpr})=$ $(0.0365,0.7308)$, and (b) the locally normalized concavity measure $Z_{\text {Local }}$ for $(\mathrm{fpr}, \mathrm{tpr})=(0.0181,0.7144)$.

\section{FEATURE COMBINATION}

An effective feature should produce a high response for vessels and a low response for all other non-vessel regions and artifacts. We see earlier that the three concavity measures ( $Z_{\text {Diff, }}, Z_{\text {Line }}$ and $\left.Z_{\text {Local }}\right)$ have different responses to different regions in a retinal image. Let $F_{\text {vess }}(\mathbf{z})$ and $F_{\text {non-vess }}(\mathbf{z})$ be the normalized vessel feature and the normalized non-vessel feature, respectively. The three concavity measures can be used to construct $F_{\text {vess }}(\mathbf{z})$ and $F_{\text {non-vess }}(\mathbf{z})$ as follows. We first normalize the three measures so that they are statistically the same. The measures $\left(\mathrm{Z}_{\text {Diff }}, \mathrm{Z}_{\text {Line }}\right.$ and $\left.\mathrm{Z}_{\text {Local }}\right)$ are assumed to follow the log-normal distribution with different means and standard derivations, which have a high occurrence near zero value and decreases dramatically [50]. The log-normal distribution property is demonstrated by the histograms given in Appendix B. In order to standardize the distributions of the three measures, statistical normalization is applied to each of the measures by first taking logarithm and then normalizing the output to zero mean and unit variance [51]. Since the response strength of $Z_{\text {Diff }}$ for vessels is the strongest among the three concavity measures, $F_{\text {vess }}(\mathbf{z})$ is taken to be equal to $Z_{\text {Diff. }}$ Since concavity measures $Z_{\text {Line }}$ and $Z_{\text {Local }}$ have the lowest response to dark lesions and noise around the bright lesions in the non-vessel region, respectively, $F_{\text {non-vess }}(\mathbf{z})$ is taken to be equal to $\min \left(Z_{\text {Line }}, Z_{\text {Local }}\right)$. The effective feature $F_{\text {eff }}(\mathbf{z})$ can then be formulated as

$$
F_{\text {eff }}(\mathbf{z})=F_{\text {vess }}(\mathbf{z}) \phi_{\text {vess }}(\mathbf{z})+F_{\text {non-vess }}(\mathbf{z}) \phi_{\text {non-vess }}(\mathbf{z})
$$

where $\phi_{\text {vess }}(\mathbf{z})$ and $\phi_{\text {non-vess }}(\mathbf{z})$ are binary indicator functions indicating the locations of vessels and non-vessel regions, respectively.

It was observed that statistically, an average of $12.7 \%$ of retina pixels in the FOV is vessels [33]. Based on the standardized normal distribution table [52], the probability for a feature having a vessel shape is given by $\operatorname{Pr}(r>1.14)=$ 0.127. A product rule from fusion theory [53] is adopted to define the vessel region $\phi_{\text {vess }}(\mathbf{z})$.

$\phi_{\text {vess }}(\mathbf{z})=B\left(Z_{\text {Diff }}>1.14\right) B\left(Z_{\text {Line }}>1.14\right) B\left(Z_{\text {Local }}>1.14\right)$

where $B(\bullet)$ is the Boolean function acting as a classifier that returns one at a pixel $\mathbf{z}$ if the corresponding expression in the bracket is true, or zero otherwise. The product rule fusion theory is a strict rule [53], where all the classifiers agree on the decision. Finally, the non-vessel region $\phi_{\text {non-vess }}(\mathbf{z})$ can be obtained by $\phi_{\text {non-vess }}(\mathbf{z})=1-\phi_{\text {vess }}(\mathbf{z})$. Figures 9 (a) and (b) show the vessel region $\phi_{\text {vess }}(\mathbf{z})$ of the retinal images having dark and bright lesions, respectively. In the next section, a lifting technique is proposed to optimize the vessel shape toward its ideal form.

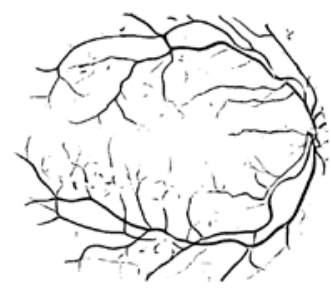

(a)

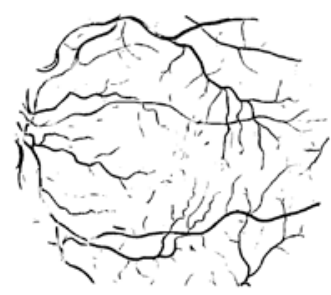

(b)
Figure 9. (a) The vessel region $\phi_{\text {vess }}(\mathbf{z})$ for the retina image in Figure 7(a). (b) The vessel region $\phi_{\text {vess }}(\mathbf{z})$ for the retina image in Figure 1(a). 


\section{LIFTING TECHNIQUE}

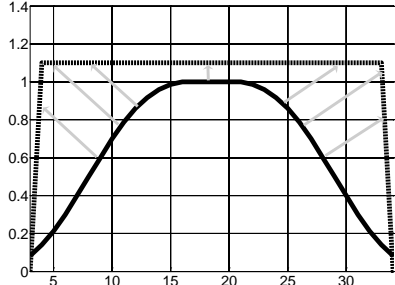

(a)

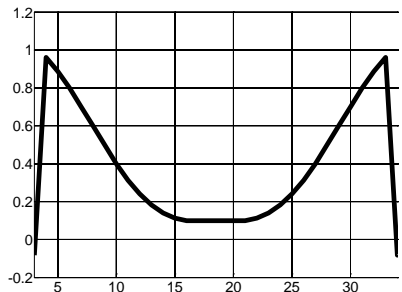

(b)
Figure 10. (a) Dash and solid lines illustrate the ideal and regularized solutions, respectively. (b) The difference between the ideal and regularized solutions.

As regularization is adopted in generating all the measures, the boundary of $F_{\text {eff }}(\mathbf{z})$ given in Equation (24) is smoothed out like the solid line shown in Figure 10(a). The dash line in Figure 10(a) illustrates the ideal solution for the vessel region. The difference between the two solutions forms a double peak shape as shown in Figure 10(b). In order to optimize the vessel shape toward its ideal solution, a lifting technique is proposed in this work. Our lifting strategy is to perform a modified diffusion process so that the vector field of $\mathrm{F}_{\text {eff }}(\mathbf{z})$ is lifted iteratively to the ideal solution. The classical diffusion equation (or heat equation) [54] is given as follows

$$
\frac{\partial u(\mathbf{z}, t)}{\partial t}=\nabla^{2} u(\mathbf{z}, t)
$$

where $u(\mathbf{z}, t)=I_{i n v}^{0}(\mathbf{z})-F_{\text {eff }}(\mathbf{z}, t)$. The inverted input image $I_{i n v}^{0}$ (taken as $-I^{0}$ ) is used as an approximation to the ideal solution. Because $I_{i n v}^{0}$ is static when the image is given, Equation (26) can be rewritten as

$$
\frac{\partial F_{\text {eff }}(\mathbf{z}, t)}{\partial t}=\nabla^{2}\left(F_{\text {eff }}(\mathbf{z}, t)-I_{i n v}^{0}(\mathbf{z})\right) .
$$

A time dimension $t \in\left[0, t_{*}\right]$ is added to $F_{\text {eff }}(\mathbf{z})$ and it is denoted as $F_{\text {eff }}(\mathbf{z}, t)$, which will be used to iteratively capture the shape difference between $F_{\text {eff }}(\mathbf{z})$ and the ideal solution. At $t=0$, $F_{\text {eff }}(\mathbf{z}, 0)=F_{\text {eff }}(\mathbf{z})$. At $t=t_{*}, F_{\text {eff }}\left(\mathbf{z}, t_{*}\right)$ is the estimated ideal solution. As the curve of $I_{i n v}^{0}$ is above $F_{\text {eff }}(\mathbf{z})$, this implies $\frac{\partial F_{\text {eff }}(\mathbf{z}, t)}{\partial t}$ for $t<t_{*}$ must be positive to allow lifting $F_{\text {eff }}(\mathbf{z})$ towards $I_{i n v}^{0}$. That is,

$$
\frac{\partial F_{\text {eff }}(\mathbf{z}, t)}{\partial t}>0 \text { for } t<t_{*} \text { and } \frac{\partial F_{\text {eff }}(\mathbf{z}, t)}{\partial t}<0 \text { for } t>t_{*}
$$

or equivalently,

$$
\frac{\partial u(\mathbf{z}, t)}{\partial t}<0 \text { for } t<t_{*} \text { and } \frac{\partial u(\mathbf{z}, t)}{\partial t}>0 \text { for } t>t_{*} .
$$

In Appendix C, we show that $F_{\text {eff }}(\mathbf{z}, t)$ reaches $I_{i n v}^{0}$ in the diffusion process for $t \geq t_{1}=g^{2}(\mathbf{m}) / 4$ with $g(\mathbf{m})=\max _{\mathbf{z}} g(\mathbf{z})=\max _{\mathbf{z}}\left\{I_{\text {inv }}^{0}(\mathbf{z})-F_{\text {eff }}(\mathbf{z}, 0)\right\}$, which can be obtained by taking the maximum among $g(\mathbf{z})$ in the vessel region $\phi_{\text {vess }}(\mathbf{z})$ (Section IV). Next, we insert an antidiffusion function $\mathrm{C}(\mathbf{z}, \mathrm{f}(t))$ to the diffusion equation so that the sign of the $\frac{\partial u(\mathbf{m}, f(t))}{\partial t}$ is positive for $t \geq t_{1}$, where $f(t)$ is a function of $t$, and the positive condition stated in Equation (29) is satisfied. The anti-diffusion function $\mathrm{C}(\mathbf{z}, t)$ is taken as $\beta g(\mathbf{m}) t^{2}$ as shown in Theorem 2 below.

Theorem 2: Assume a single peak shape follows a Gaussian distribution with mean $\mathbf{m}$ and variance $t_{0}>0$. The modified diffusion

process $\frac{\partial u(\mathbf{z}, f(t))}{\partial f(t)}=\nabla^{2} u(\mathbf{z}, f(t))+\beta g(\mathbf{m}) f^{2}(t)>$

0 when $f(t) \geq 1$ at $\mathbf{z}=\mathbf{m}$ and $\beta=\frac{1}{4 \pi}$.

The proof of theorem 2 is given in Appendix D. In the theorem, if we take $f(t)=t / t_{1}$ and apply the chain rule [54], the sign of $\frac{\partial u(\mathbf{m}, f(t))}{\partial t}$ will be positive for $t \geq t_{1}$. Also, the theorem is still valid for very small $t_{0}$, for which the peak shape is in the form of a delta-function. This means our algorithm still performs lifting even if the vessel region of $\mathrm{F}_{\text {eff }}(\mathbf{z})$ is closely beneath the $I_{i n v}^{0}$. Finally, a vector field matching function $M(\mathbf{z}, t)=\frac{1}{2}\left(1-\frac{\nabla F_{\text {eff }}(\mathbf{z}, t) \bullet \nabla I_{i n v}^{0}(\mathbf{z})}{\left\|\nabla F_{\text {eff }}(\mathbf{z}, t)\right\|\left\|\nabla I_{i n v}^{0}(\mathbf{z})\right\|}\right) \quad$ is imposed onto the modified diffusion process as

$$
\frac{\partial u(\mathbf{z}, f(t))}{\partial f(t)}=\nabla^{2} u(\mathbf{z}, f(t))+M(\mathbf{z}, f(t)) \beta g(\mathbf{m}) f^{2}(t) \text {. }
$$

$M(\mathbf{z}, f(t))$ measures the degree of shape similarity between $\mathrm{F}_{\text {eff }}(\mathbf{z}, \mathrm{f}(t))$ and $I_{i n v}^{0}$ by their vector fields. If both shapes at $\mathbf{z}$ are not matched, the two vectors will be in opposite directions and $M(\mathbf{z}, f(t))=1$. Otherwise, it is zero. In real application, the single peak shape may not strictly follow the Gaussian distribution as assumed by Theorem 2. The role of $M(\mathbf{z}, f(t))$ is to allow lifting in the modified diffusion process while preserving the shape similarity between $\mathrm{F}_{\text {eff }}(\mathbf{z}, f(t))$ and $I_{i n v}^{0}$. For the pixels having low shape similarity, $M(\mathbf{z}, f(t))=1$ and it will penalize lifting in Equation (30) for $f(t) \geq 1$. For the pixels having high shape similarity, $M(\mathbf{z}, f(t))=0$ and further lifting would proceed. By substituting $u(\mathbf{z}, f(t))=I_{\text {inv }}^{0}(\mathbf{z})-F_{\text {eff }}(\mathbf{z}, f(t))$ into Equation (30), we have

$\frac{\partial F_{e f f}(\mathbf{z}, f(t))}{\partial f(t)}=\nabla^{2}\left(F_{e f f}(\mathbf{z}, f(t))-I_{i n v}^{0}(\mathbf{z})\right)-M(\mathbf{z}, f(t)) \beta g(\mathbf{m}) f^{2}(t)$. 
Now, the time variable $f(t)=t / \frac{g^{2}(\mathbf{m})}{2}$ is converted to time $t$ by the chain rule [54]:

$$
\begin{aligned}
\frac{\partial F_{\text {eff }}(\mathbf{z}, f(t))}{\partial t} & =\frac{\partial F_{\text {eff }}(\mathbf{z}, f(t))}{\partial f(t)} \frac{d f(t)}{d t} \\
& =\left\{\nabla^{2}\left(F_{\text {eff }}(\mathbf{z}, f(t))-I_{\text {inv }}^{0}(\mathbf{z})\right)-M(\mathbf{z}, f(t)) \beta g(\mathbf{m}) f^{2}(t)\right\} \frac{d f(t)}{d t}
\end{aligned}
$$

where $\frac{d f(t)}{d t}=\gamma=1 / \frac{g^{2}(\mathbf{m})}{2}$. The solution of Equation (32) can be obtained numerically by discretizing the time variable of the term $\frac{\partial F_{\text {eff }}(\mathbf{z}, f(t))}{\partial t}$ with a step size of $\xi$ (which is taken as 0.5 in our experiments). The implementation is given in Table 2. Figures 11(a) and 11(d) show the difference between $F_{\text {eff }}\left(\mathbf{z}, \mathrm{t}_{*}\right)$ and $F_{\text {eff }}(\mathbf{z}, 0)$, in which darker color represents more lifting yielded by the technique. Figures 11(b) and (e) show the segmentation results using the proposed method, where the final segmentation is done by thresholding $F_{\text {eff }}(\mathbf{z})$ with a threshold chosen such that the false positive rate (fpr) is 0.023 . In the next section, experiments will be conducted to evaluate the performance of the proposed method in comparison with the state-of-the-art techniques.

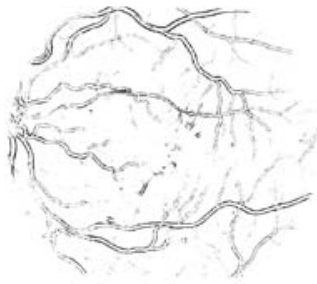

(a)

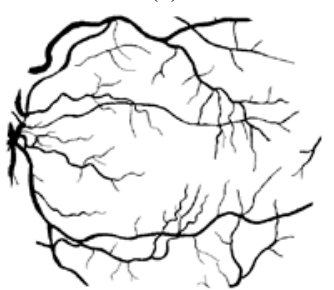

(c)

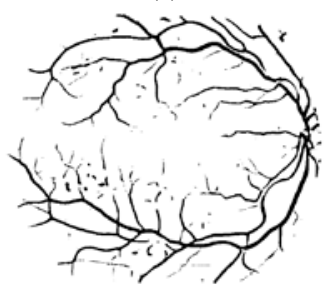

(e)

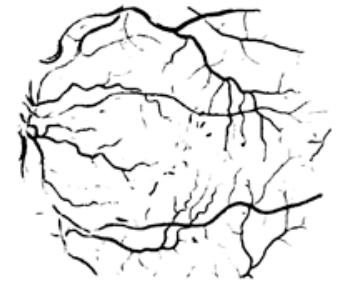

(b)

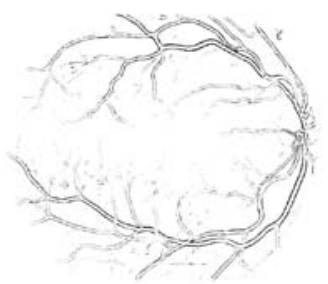

(d)

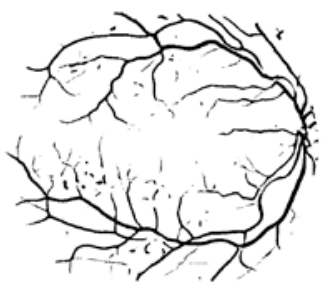

(f)
Figure 11. Lifting and segmentation results in the proposed system on retinas having dark and bright lesions respectively. (a) The strength of $F_{\text {eff }}\left(\mathbf{z}, \mathrm{t}_{*}\right)$ $-F_{\text {eff }}(\mathbf{z}, 0)$ on the retinal image shown in Figure 1(a). (b) Final result of the proposed method $(f p r, t p r)=(0.0234,0.7450)$. (c) Ground truth 1 . (d) The

strength of $F_{\text {eff }}\left(\mathbf{z}, \mathrm{t}_{*}\right)-F_{\text {eff }}(\mathbf{z}, 0)$ on the retinal image shown in Figure 7(a). (e) Final result of the proposed method $(f p r, t p r)=(0.0230,0.7864)$. (f) Ground truth 1.

\section{Table 2. Lifting technique}

Step 0: Normalize $F_{\text {eff }}$ and $I_{i n v}^{0}$ to the same range by subtracting their minimum values and then multiply $I_{i n v}^{0}$ by a constant $s=\arg \min \left\|F_{\text {eff }}(\mathbf{z}, 0)-s I_{i n v}^{0}\right\|^{2}$. Set $t=0, F_{\text {eff }}(\mathrm{z}, 0)=F_{\text {eff }}$ and $I_{t}=I_{i n v}^{0}$. Compute $\gamma=1 / \frac{g^{2}(\mathbf{m})}{2}$;

Step 1: $t=t+\xi$

For the $\mathbf{z}$ th pixel, do

$\begin{array}{llll}\text { Step } & \text { 2: } & \text { Compute }(t)=t \gamma \quad \text { and } & \Gamma_{\mathrm{t}}(\mathbf{z})\end{array}$

$=\nabla^{2}\left(F_{\text {eff }}(\mathbf{z}, t)-I_{\text {inv }}^{0}(\mathbf{z})\right)-M(\mathbf{z}, t) \beta g(\mathbf{m}) f^{2}(t)$.

Step 3: If $\Gamma_{\mathrm{t}}(\mathbf{z})>0, F_{\text {eff }}(\mathbf{z}, t+\xi)=F_{\text {eff }}(\mathbf{z}, t)+\Gamma_{\mathrm{t}}(\mathbf{z}) \gamma \xi$.

Otherwise, $F_{\text {eff }}(\mathbf{z}, t+\xi)=F_{\text {eff }}(\mathbf{z}, t)$.

End do

Step 4: If $\sqrt{\sum_{\mathbf{z} \in \Omega}\left|F_{\text {eff }}(\mathbf{z}, t+\xi)-F_{\text {eff }}(\mathbf{z}, t)\right|^{2}}>\varepsilon$, go to step $1 . \Omega$ here is the image domain. Otherwise, terminate and output $F_{\text {eff }}\left(\mathbf{z}, t_{*}\right)=F_{\text {eff }}(\mathbf{z}, t)$.

\section{EXPERIMENT}

A. Databases: The performance of the proposed method is evaluated on two publicly available databases, STARE and DRIVE, which are widely used by most existing methods. The STARE $^{1}$ database contains 20 images with 10 pathological and 10 normal retinal images. Each of the images has a size of $700 \times 605$ pixels with 8 bits per color channel. The DRIVE ${ }^{2}$ database consists of 40 images, which are divided into training and test sets with 20 images in each. The retinal images in DRIVE databases are mainly healthy with some of them containing a few mild lesions. Both databases provide two ground truths manually marked by two independent observers. All the results presented in this paper are obtained by using the first human observer in the database as ground truth.

B. Experimental Evaluation: Three measures are used for performance evaluation. The accuracy and the receiver operating characteristic (ROC) curve are used as evaluation measures in the same way as used by existing methods [37, 39]. The performance in the pathological region (PUR) [41] is also used as a measure for evaluating the performance on pathological regions of a given retinal image. The accuracy on a retinal image is defined as the number of true positive vessel pixels plus the number of true positive non-vessel pixels divided by the total number of pixels in the FOV. The ROC is constructed by the true positive rate (tpr) as the vertical axis and the false positive rate (fpr) as the horizontal axis [55]. The closer the ROC curve approaches the top left corner, the better the performance of the method. A common single measure to quantify the performance of a method is to compute the area $\mathrm{R}_{\text {area }}$ under the ROC curve. An area closer to one means a better performance. The PUR is defined as the number of true positive pixels plus the number of true positive non-vessel pixels that are at least $\mathrm{T}$ pixels (which ranges from 0 to 25 as in [41]) away from the true positive blood vessels pixels,

\footnotetext{
${ }^{1}$ http://www.parl.clemson.edu/stare/probing/

${ }^{2}$ http://www.isi.uu.nl/Research/Databases/DRIVE/
} 
divided by the total number of pixels in FOV. A larger PUR indicates a better performance of the method.

The green channel of a retinal image is used as input because it is often adopted in the research community [34, 37, 39] and has been shown empirically to give the best segmentation results. To reduce the artifacts produced near the border of the camera aperture by a segmentation method, a border extension pre-processing technique [37] is utilized. Figure 12 is an example of the preprocessing result. The region inside the green contour is the green channel image while the outside non-dark region is the extended pixels generated by the inside pixels.

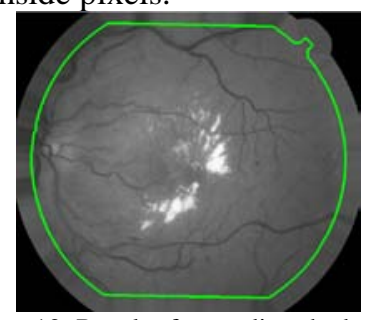

Figure 12. Result of extending the border.

C. Results: Tables 3, 4 and 5 show the results of the proposed method together with the benchmark methods obtained on the complete STARE database, the complete DRIVE database and the abnormal images in the STARE database, respectively. We also study the effect of the perceptive transform. The results with the transform (denoted as Proposed Method) and without the transform, which is in the original image space (denoted as Proposed Method (ImS)), are shown in the tables. The comparative results of the benchmark methods in the tables are obtained from [34-35, 37-41]. For the DRIVE database, there are two different results for the line detector method as reported in [34] and [35]. Both of them are shown in Table 4 for reference together with the result from our own implementation of the line detector algorithm of [35], denoted as Line (Impl.). Ideally, the results of Line (Impl.) should be identical to the results of [35]. However, we were unable to achieve the results reported in [35] due to unknown reason. Line (Impl.) is also reported in Tables 3 and 5. The results of another state-of-the-art method, the supervised learning method proposed by Soares et al.[39], are available on their website $^{3}$. It consists of two sets of results obtained by two implementations of their method, which are trained by STARE and DRIVE databases respectively. They are denoted as Soares et al. (STARE) and Soares et al. (DRIVE) in this paper.

Table 3 shows the results on the complete STARE database. The proposed unsupervised method achieves the highest $R_{\text {area }}$ among all the methods including the three supervised learning methods. The accuracy of the proposed method is among the best and even outperforms the human observer. Figure 13(a) shows the ROC curves of the proposed method and the supervised learning methods. The proposed method is completely above the other two methods and passes through the (fpr, tpr) of the human observer. Table 4 shows the results on the complete DRIVE database. The proposed method and the method of Soares et al. (DRIVE) yield the largest $\mathrm{R}_{\text {area }}$ compared to the others. The accuracy of the proposed method is also among the best. Figure 13(b) shows the ROC curves of the proposed method and the two supervised learning systems. The curves obtained by the proposed method and the method of Soares et al. (DRIVE) are almost the same. But the proposed method is closer to the $2^{\text {nd }}$ observer than Soares et al. (DRIVE). Table 5 shows the results on the abnormal images in the STARE database. Both the accuracy and $R_{\text {area }}$ of the proposed method are the best among all the unsupervised and supervised learning methods. The $\mathrm{R}_{\text {area }}$ of the proposed method is larger than the second best method by 0.01 . The method of Lam and Yan [41] is specially designed to handle abnormal retinas. However, our method still outperforms it by nearly 0.01 in accuracy and 0.03 in $R_{\text {area }}$, respectively. Figure 13(c) shows the ROC curves of the proposed method, the two supervised learning methods, and the method of Lam and Yan [41]. The proposed method is able to yield the ROC curve significantly above all the other methods and even above the human observer, which is not achieved by any of the state-ofthe-art benchmark methods. Figure 14 shows the overall PUR measure for the abnormal retinas in the STARE database. The proposed method is the best and its PUR curve is completely above those of the other methods.

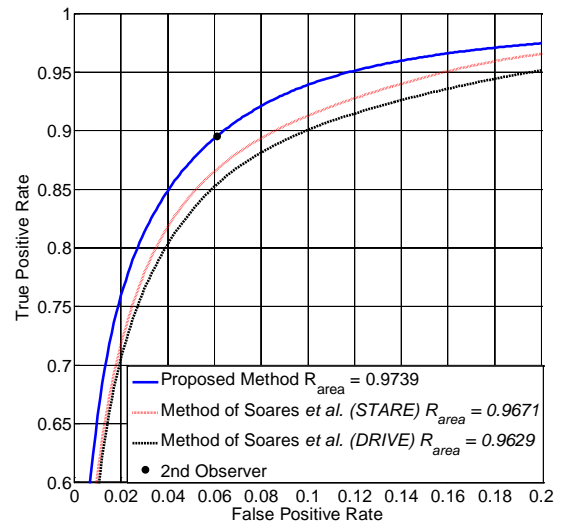

(a)

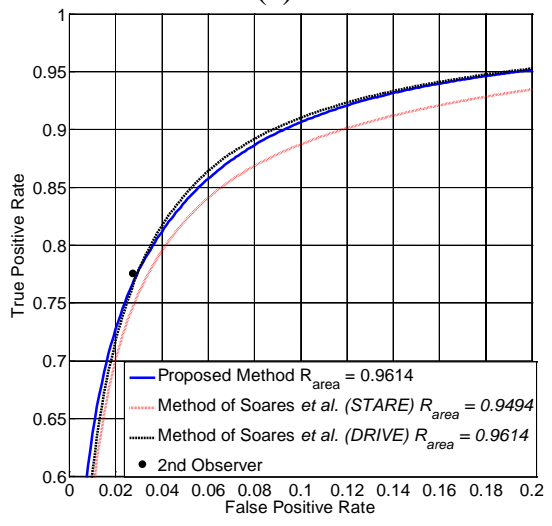

(b)

\footnotetext{
${ }^{3}$ http://retina.incubadora.fapesp.br/portal/downloads/results
} 


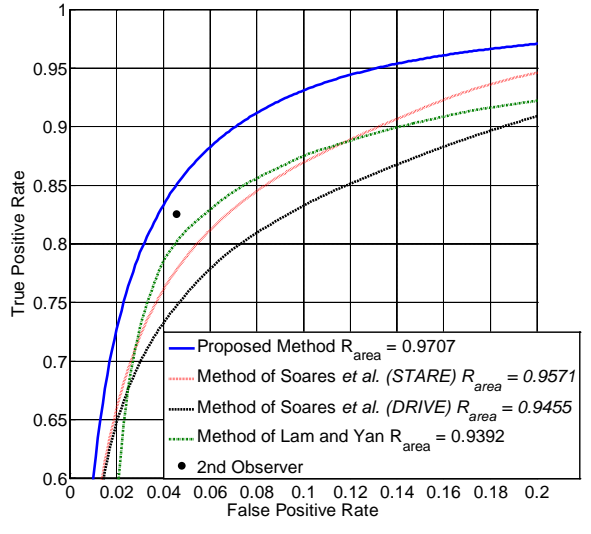

(c)

Figure 13. The ROC curves obtained by the proposed method, the two supervised systems [39] and the method of Lam and Yan [41] on (a) the complete STARE database, (b) the complete DRIVE database and (c) the

pathological retinal images only in the STARE database. The dark dot indicates the true and false positive rates of the second human observer given in the database.

Table 3. Results on the complete STARE database.

\begin{tabular}{|c|c|c|}
\hline STARE & Accuracy & $\mathrm{R}_{\text {area }}$ \\
\hline \multicolumn{3}{|c|}{ Unsupervised Methods } \\
\hline Jiang and Mojon [33] & 0.9009 & 0.9298 \\
\hline Hoover et al. [32] & 0.9275 & 0.7590 \\
\hline Line (Impl.) & 0.9422 & 0.9615 \\
\hline Line (TMI) [34] & 0.9584 & 0.9602 \\
\hline Mendonça and Campilho [40] & 0.9479 & N/A \\
\hline Proposed Method (ImS) & 0.9454 & 0.9562 \\
\hline Proposed Method & 0.9567 & 0.9739 \\
\hline \multicolumn{3}{|c|}{ Supervised Learning Methods } \\
\hline Staal et al. [37] & 0.9516 & 0.9614 \\
\hline Soares et al. (DRIVE) [39] & 0.9469 & 0.9629 \\
\hline Soares et al. (STARE) [39] & 0.9480 & 0.9671 \\
\hline $\begin{array}{l}\text { 2nd Observer from the } \\
\text { database }\end{array}$ & 0.9351 & N/A \\
\hline
\end{tabular}

Table 4. Results on the complete DRIVE database.

\begin{tabular}{|c|c|c|}
\hline STARE & Accuracy & $\mathrm{R}_{\text {area }}$ \\
\hline \multicolumn{3}{|c|}{ Unsupervised Methods } \\
\hline Jiang and Mojon [33] & 0.8911 & 0.9327 \\
\hline Line (C\&S) [35] & 0.9261 & 0.9348 \\
\hline Line (Impl.) & 0.9320 & 0.9410 \\
\hline Line (TMI) [34] & 0.9563 & 0.9558 \\
\hline Mendonça and Campilho [40] & 0.9463 & N/A \\
\hline Proposed Method (ImS) & 0.9383 & 0.9519 \\
\hline Proposed Method & 0.9472 & 0.9614 \\
\hline \multicolumn{3}{|c|}{ Supervised Learning Methods } \\
\hline Matched filter k-NN [38] & 0.9416 & 0.9294 \\
\hline Staal et al. [37] & 0.9441 & 0.9520 \\
\hline Soares et al. (DRIVE) [39] & 0.9466 & 0.9614 \\
\hline Soares et al. (STARE) [39] & 0.9445 & 0.9494 \\
\hline $\begin{array}{c}\text { 2nd Observer from the } \\
\text { database }\end{array}$ & 0.9473 & N/A \\
\hline
\end{tabular}
database

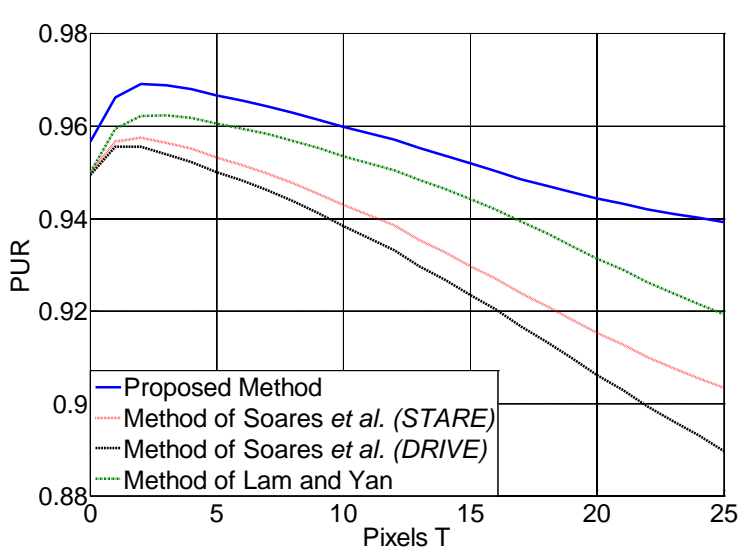

Figure 14. The performance evaluation measure PUR for different methods using all the pathological images.

Table 5. Results on the abnormal retinas only in the STARE database.

\begin{tabular}{|c|c|c|}
\hline STARE & Accuracy & R $_{\text {area }}$ \\
\hline \hline \multicolumn{1}{|c|}{ Unsupervised Methods } \\
\hline Vermeer et al. [56] & 0.9287 & 0.9187 \\
\hline Jiang and Mojon [33] & 0.9337 & 0.8906 \\
\hline Line (Impl.) & 0.9352 & 0.9343 \\
\hline Mendonça and Campilho [40] & 0.9426 & N/A \\
\hline Lam and Yan [41] & 0.9474 & 0.9392 \\
\hline Proposed Method (ImS) & 0.9435 & 0.9460 \\
\hline Proposed Method & 0.9556 & 0.9707 \\
\hline \hline \multicolumn{2}{|c|}{ Supervised Learning Methods } \\
\hline Soares et al. (DRIVE) [39] & 0.9428 & 0.9455 \\
\hline Soares et al. (STARE) [39] & 0.9425 & 0.9571 \\
\hline $\begin{array}{c}\text { 2nd Observer from the } \\
\text { database }\end{array}$ & 0.9410 & N/A \\
\hline
\end{tabular}

Table 6. Running Times for Different Methods.

\begin{tabular}{|c|c|c|c|}
\hline & Time & PC & Software \\
\hline Line (Impl.) & $30 \mathrm{sec}$ & $\begin{array}{c}\text { Duo CPU 1.83 } \\
\text { GHz, 2.0 Gb of } \\
\text { RAM }\end{array}$ & Matlab \\
\hline $\begin{array}{c}\text { Mendonça and } \\
\text { Campilho [40] }\end{array}$ & 2.5 to 3 mins & $\begin{array}{c}\text { Pentium-4 PC, } \\
\text { 3.2 GHz, 960 } \\
\text { Mb of RAM }\end{array}$ & Matlab \\
\hline Soares et al. [39] & $\begin{array}{c}\text { 3 mins (9hrs for } \\
\text { training) }\end{array}$ & $\begin{array}{c}\text { PC (2167 MHz } \\
\text { clock) with 1- } \\
\text { GB memory. }\end{array}$ & Matlab \\
\hline Lam and Yan & [41] mins & $\begin{array}{c}\text { Duo CPU 1.83 } \\
\text { GHz, 2.0 Gb of } \\
\text { RAM }\end{array}$ & Matlab \\
\hline Proposed Method & 13 mins & $\begin{array}{c}\text { Duo CPU 1.83 } \\
\text { GHz@, 2.0 Gb } \\
\text { of RAM }\end{array}$ & Matlab \\
\hline Staal et al. [37] & 15 mins & $\begin{array}{c}\text { Pentium-III PC, } \\
\text { running at 1.0 } \\
\text { GHz with 1-GB } \\
\text { memory }\end{array}$ & N/A \\
\hline
\end{tabular}

In Tables 3, 4 and 5, we observe that there is an improvement of around 0.01 to 0.02 in both accuracy and $R_{\text {area }}$ when perceptive transform is used. This is in accordance with the argument of Mendonça and Campilho [40] that the geometric operation is more robust to noise than the arithmetic operation. In this work, the arithmetic operations in the perceptive space are equivalent to geometric operations in the original image space: 


$$
\frac{1}{N} \sum_{k=1}^{N} \ln \left(x_{k}\right)=\ln \left[\prod_{k=1}^{N}\left(x_{k}\right)\right]^{\frac{1}{N}} .
$$

The running times of different methods are given in Table 6 where the running times of the methods of Mendonça and Campilho [40], Soares et al. [39] and Staal et al. [37] are obtained from their papers. The proposed method takes on average 13 mins to get a segmentation result. Most of the time is spent on computing $C_{\mathrm{SD}}$ and $C_{\mathrm{SI}}$. Boosting up the convergence rates of the two widely adopted TV and MS models can significantly shorten the running time and this is our future work. Also in real applications, the computation time can be significantly reduced by implementing the algorithm in $\mathrm{C} / \mathrm{C}++$ programming.

\section{CONCLUSION}

This paper presents a novel regularization-based multiconcavity approach for effectively segmenting blood vessels in both normal and pathological retinas with bright and dark lesions in a single system. A perceptive transform derived from Weber's law is proposed to map an input image into a perceptive space for robust vessel segmentation. Our results show that system performances of both accuracy and $R_{\text {area }}$ tested on grey-scale intensity, RGB and $\mathrm{L}^{*} \mathrm{a} * \mathrm{~b} *$ color channels are consistently better in the perceptive space than in the image space, demonstrating the broader effectiveness of the proposed perceptive space method. As the bright lesion has a steep intensity transition profile, measuring the degree of concavity can effectively distinguish the bright lesions from the vessels and non-vessels. Dark lesions have an irregular shape intensity structure while blood vessels have a line-shape intensity structure. The line-shape concavity is modeled to prune the dark lesions while preserving the regions of blood vessels. The relative intensities of noise in different parts of the retinal image are different due to the fact that a retinal image has a spherical intensity variation. A locally normalized concavity scheme is proposed to normalize the strengths of noise removal in different regions. The lifting scheme is designed to perform a modified diffusion process ensuring the detected vessel shape is optimized iteratively to the ideal solution.

In contrast to the existing methods, the proposed method can identify vessels in pathological retinal images with both bright and dark lesions. More importantly, it can work simultaneously for health and pathological retinas. Extensive experimental results on STARE and DRIVE databases demonstrated the superior performance of proposed method on both healthy and abnormal retinas. As an interesting future work, the proposed techniques may be extended to solve the challenging problem of lesion detection.

\section{ACKNOWLEDGMENT}

The authors would like to thank Staal et al. and Hoover et al. for their DRIVE and STARE databases used in this research and the anonymous reviewers for their suggestions and comments, which have greatly improved the quality of this paper.

\section{REFERENCES}

[1] The Eye Diseases Prevalence Research Group, "Prevalence of Age Related Macular Degeneration in the United States," Arch. Ophth., Vol. 122, pp. 564-572, 2004.

[2] The Eye Diseases Prevalence Research Group, "The Prevalence of Diabetic Retinopathy Among Adults in the United States," Arch. Ophth., Vol. 122, no. 4, pp. 552-563, 2004.

[3] The Eye Diseases Prevalence Research Group, "Prevalence of Open Angle Glaucoma Among Adults in the United States," Arch. Ophth., Vol. 122, no. 4, pp. 532-538, 2004.

[4] Springhouse, Professional Guide to Diseases, Lippincott Williams \& Wilkins; Seventh Edition edition, 2001

[5] J. A. Shoemaker, Vision problems in the U.S. Tech. Rep. U.S. National Institute of Health, 2002.

[6] A. A. Cavallerano, J. D. Cavallerano, P. Katalinic, A. M. Tolson, and L. P. Aiello, "Use of Joslin Vision Network Digital-video Nonmydriatic Retinal Imaging to Assess Diabetic Retinopathy in a Clinical Program," Retina, vol. 23, pp. 215-223, April 2003.

[7] S. R. Fransen, T. C. Leonard-Martin, W. J. Feuer, and P. L. Hildebrand,

" Clinical Evaluation of Patients with Diabetic Retinopathy: Accuracy of the Inoveon Diabetic Retinopathy-3DT System,” Ophthalmology, Vol. 109, pp. 595-601, Mar. 2002.

[8] M. Niemeijer, B. van Ginneken, J. Staal, M. Suttorp-Schulten and M. Abramoff, "Automatic Detection of Red Lesions in Digital Color Fundus Photographs," IEEE Transactions on Medical Imaging, Vol. 24, pp. 584-592, 2005.

[9] N. Patton, T. Aslam, T. MacGillivray, I. Deary, B. Dhillon, R. Eikelboom, K. Yogesan and I. Constable, "Retinal Image Analysis: Concepts, Applications and Potential," Progress in Retinal and Eye Research, Vol. 25, pp. 99-127, 2006.

[10] M. Niemeijera, M. Abràmoffb and B. van Ginnekena, "Image Structure Clustering for Image Quality Verification of Color Retina Images in Diabetic Retinopathy Screening," Medical Image Analysis, Vol. 10, pp. 888-898, 2006,

[11] A. Mendonça, A. Campilho and J. Nunes, "Automatic Segmentation of Microaneurysms in Retinal Angiograms of Diabetic Patients," International Conference on Image Analysis and Processing, pp. 728733, 1999.

[12] A. Osareh, M. Mirmehdi, B. Thomas and R. Markham, "Automated Identification of Diabetic Retinal Exudates in Digital Colour Images," British Journal of Ophthalmology, Vol. 87, pp. 1220-1223, 2003.

[13] L. Huiqi and O. Chutatape, "Automated Feature Extraction in Color Retinal Images by a Model based Approach," IEEE Transactions on Biomedical Engineering, Vol. 51, pp. 246-254, 2004.

[14] J. Lowell, A. Hunter, D. Steel, A. Basu, R. Ryder, and R. Kennedy, "Measurement of Retinal Vessel Widths from Fundus Images Based on 2-D Modeling," IEEE Transactions on Medical Imaging, Vol. 23, pp. 1196-1204, 2004.

[15] A. Youssif, A. Ghalwash and A. Ghoneim, "Optic Disc Detection From Normalized Digital Fundus Images by Means of a Vessels' Direction Matched Filter," IEEE Transactions on Medical Imaging, Vol. 27, pp. 11-18, 2008.

[16] K. Tobin, E. Chaum, V. Govindasamy and T. Karnowski, "Detection of Anatomic Structures in Human Retinal Imagery," IEEE Transactions on Medical Imaging, Vol. 26, pp. 1729-1739, 2007.

[17] E. Grisan and A. Ruggeri, "Segmentation of Candidate Dark Lesions in Fundus Images Based on Local Thresholding and Pixel Density," Annual International Conference of the IEEE Engineering in Medicine and Biology Society, pp. 6736-6739, 2007.

[18] M. Larsen, J. Godt, N. Larsen, H. Lund-Andersen, A. Katrin Sjølie, E. Agardh, H. Kalm, M. Grunkin and D. Owens, "Automated Detection of Fundus Photographic Red Lesions in Diabetic Retinopathy," Investigative Ophthalmology \& Visual Science, Vol. 44, pp. 761-766, 2003

[19] C. Sáncheza, R. Horneroa, M. Lópezb, M. Aboyc, J. Pozaa and D. Abásoloa, "A Novel Automatic Image Processing Algorithm for Detection of Hard Exudates Based on Retinal Image Analysis," Medical Engineering \& Physics, Vol. 30, pp. 350-357, 2008. 
[20] G. Quellec, M. Lamard, P.M. Josselin, G. Cazuguel, B. Cochener and C. Roux, "Optimal Wavelet Transform for the Detection of Microaneurysms in Retina Photographs", IEEE Transactions on Medical Imaging, Vol. 27, pp. 1230-1241, 2008.

[21] M. Sofka and C. V. Stewart, "Retinal Vessel Centerline Extraction Using Multiscale Matched Filters, Confidence and Edge Measures,” IEEE Transactions on Medical Imaging, Vol. 25, pp. 1531-1546, 2006.

[22] M. Sofka and C. V. Stewart, Erratum to "Retinal Vessel Centerline Extraction Using Multiscale Matched Filters, Confidence and Edge Measures,” IEEE Transactions on Medical Imaging, Vol. 26, No. 1, pp. 133-133, 2007.

[23] Y. Sun, "Automated Identification of Vessel Contours in Coronary Arteriograms by an Adaptive Tracking Algorithm," IEEE Trans Med Imaging. Vol. 8, pp. 78-88, 1989.

[24] F. Zana and J. Klein "A Multimodal Registration Algorithm of Eye Fundus Images Using Vessels Detection and Hough Transform,” IEEE Transactions On Medical Imaging, Vol. 18, pp.: 419-428, 1999.

[25] E. Grisan, M. Foracchia, and A. Ruggeri. "A Novel Method for the Automatic Grading of Retinal Vessel Tortuosity,” IEEE Transactions on Medical Imaging, Vol. 27, pp. 310-319, 2008.

[26] M. Niemeijer, M. Abramoff and B. van Ginneken, "Segmentation of the Optic Disc, Macula and Vascular Arch in Fundus Photographs," IEEE Transactions on Medical Imaging, Vol. 26, pp. 116-127, 2007.

[27] A. Fleming, S. Philip, K. Goatman, J. Olson and P. Sharp, "Automated Microaneurysm Detection Using Local Contrast Normalization and Local Vessel Detection," IEEE Transactions on Medical Imaging, Vol. 25, pp. 1223-1232, 2006.

[28] C. I. Sanchez, R. Hornero, M. I. Lopez and J. Poza, "Retinal Image Analysis to Detect and Quantify Lesions Associated with Diabetic Retinopathy," Proc. 26th IEEE Annual International Conf. on Engineering in Medicine and Biology Society (EMBC), Vol. 3, pp. 1624-1627, 2004.

[29] M. Niemeijer, B. van Ginneken, S. Russell, M. Suttorp-Schulten, and M. Abràmoff, "Automated Detection and Differentiation of Drusen, Exudates, and Cotton-Wool Spots in Digital Color Fundus Photographs for Diabetic Retinopathy Diagnosis," Investigative Ophthalmology and Visual Science, Vol. 48, pp. 2260-2267, 2007

[30] L. Gang, C. Opas, L. Huiqi and K.Shankar, "Abnormality Detection in Automated Mass Screening System of Diabetic Retinopathy," Proceedings of the Fourteenth IEEE Symposium on Computer-Based Medical Systems, pp. 132-137, 2001.

[31] S. Chaudhuri, S. Chatterjee, N. Katz, M. Nelson, and M. Goldbaum, "Detection of Blood Vessels in Retinal Images Using Two-dimensional Matched Filters,” IEEE Transactions on Medical Imaging, Vol. 8, pp. 263-269, 1989.

[32] A. Hoover, V. Kouznetsova, and M. Goldbaum, "Locating Blood Vessels in Retinal Images by Piece-wise Threshold Probing of a Matched Filter Response,” IEEE Transactions on Medical Imaging, Vol. 19, pp. 203-210, 2000.

[33] X. Jiang and D. Mojon, “Adaptive Local Thresholding by Verificationbased Multithreshold Probing with Application to Vessel Detection in Retinal Images,” IEEE Transactions on Pattern Analysis and Machine Intelligence, Vol. 25, pp. 131-137, 2003.

[34] E. Ricci and R. Perfetti, "Retinal Blood Vessel Segmentation Using Line Operators and Support Vector Classification,” IEEE Transactions on Medical Imaging, Vol. 26, pp. 1357-1365, 2007.

[35] R. Perfetti, E. Ricci, D. Casali and G. Constantini, "Cellular Neural Networks With Virtual Template Expansion for Retinal Vessel Segmentation,” IEEE Transactions on Circuits and Systems II: Express Briefs, Vol. 54, pp. 141-145, 2007.

[36] Y. Tolias and S. Panas, "A Fuzzy Vessel Tracking Algorithm for Retinal Images Based on Fuzzy Clustering," IEEE Transactions on Medical Imaging, Vol. 17, pp.263-73, 1998.

[37] J. Staal, M. D. Abramoff, M. Niemeijer, M. A. Viergever, and B. van Ginneken, "Ridge Based Vessel Segmentation in Color Images of the Retina,” IEEE Transactions on Medical Imaging, Vol. 23, pp.501-509, 2004.

[38] M. Niemeijer, J. Staal, B. van Ginneken, M. Loog, and M. D. Abramoff, "Comparative Study of Retinal Vessel Segmentation
Methods on a New Publicly Available Database,” in SPIE Med. Imag., J.M. Fitzpatrick and M. Sonka, Eds., Vol. 5370, pp. 648-656, 2004.

[39] J. V. B. Soares, J. J. G. Leandro, R. M. Cesar Jr., H. F. Jelinek and M. J. Cree, "Retinal Vessel Segmentation Using the 2-D Gabor Wavelet and Supervised Classification," IEEE Transactions on Medical Imaging, Vol. 25, No. 9, pp.1214-1222, 2006.

[40] A. M. Mendonça and A. Campilho, "Segmentation of Retinal Blood Vessels by Combining the Detection of Centerlines and Morphological Reconstruction,” IEEE Transactions on Medical Imaging, Vol. 25, pp.1200- 1213, 2006.

[41] B. S. Y. Lam and H. Yan, “A Novel Vessel Segmentation Algorithm for Pathological Retina Images Based on the Divergence of Vector Fields,” IEEE Transactions on Medical Imaging, Vol. 27, pp. 237-246, 2008.

[42] E. Cartereette and M. Friedman, Handbook of Perception, Vol. 5, New York: Academic Press, 1975.

[43] Jianhong Shen and Yoon-Mo Jung, "Weberized Mumford-Shah Model with Bose-Einstein Photon Noise,” Appl. Math. Optim., Vol. 53, pp. 331-358, 2006.

[44] T. Lindeberg, "Edge Detection and Ridge Detection with Automatic Scale Selection," IEEE Conf. on Computer Vision and Pattern Recognition, CVPR'96, pp. 465-470, 1996.

[45] D. Mumford and J. Shah, "Optimal Approximations by Piecewise Problems,” Comm. Pure Applied. Math., Vol. 42, pp. 577-685, 1989.

[46] T. Chan and J. Shen, Image Processing and Analysis: Variational, PDE, Wavelet, and Stochastic Methods, Philadelphia: Society for Industrial and Applied Mathematics, 2005.

[47] T. Chan, S. Osher and J. Shen, "The Digital TV Filter and Nonlinear Denoising," IEEE Transactions on Image Processing, Vol. 10, No. 2, pp. 231-241, 2001.

[48] R. Zwiggelaar, S.M. Astley, C. R. M. Boggis, and C. J. Taylor, "Linear Structures in Mammographic Images: Detection and Classification,” IEEE Transactions on Medical Imaging, Vol. 23, pp. 1077-1086, 2004.

[49] J. Bezdek, Pattern Recognition with Fuzzy Objective Function Algorithms, Plenum Pub Corp, 1981

[50] I.N. Bronshtein, K.A. Semendyayev, G. Musiol, H. Muehlig and H. Mühlig, Handbook of Mathematics, Springer; 4th edition, 2004.

[51] M. Law, M. A. T. Figueiredo and A. K. Jain. "Simultaneous Feature Selection and Clustering Using Mixture Models", IEEE Transactions of Pattern Analysis and Machine Intelligence, Vol. 26, No. 9, pp. 11541166, 2004.

[52] D. P. Bertsekas and J. N. Tsitsiklis, Introduction to Probability, 2nd Edition, American Mathematical Society, 1998.

[53] J. Kittler, M. Hatef, R. Duin, J. Matas, "On Combining Classifiers," IEEE Transactions on Pattern Analysis and Machine Intelligence, Vol. 20, pp. 226-239, 1998.

[54] W. Kaplan, Advanced calculus, 4th edition, Publisher Reading, Mass.: Addison-Wesley, 1991.

[55] N. A. Obuchowski, "Receiver Operating Characteristic Curves and Their Use in Radiology,” Radiology, Vol. 229, pp. 3-8, 2003.

[56] K. A. Vermeer, F. M. Vos, H. G. Lemij and A. M. Vossepoel, “A Model Based Method for Retinal Blood Vessel Detection," Computers in Biology and Medicine, Vol. 34, pp. 209-219, 2004. 